\title{
Differential specificity between closely related corals and abundant Endozoicomonas endosymbionts across global scales
}

\author{
Matthew J Neave ${ }^{1,2}$, Rita Rachmawati ${ }^{3}$, Liping Xun ${ }^{2}$, Craig T Michell ${ }^{1}$, David G Bourne ${ }^{4}$, \\ Amy Apprill ${ }^{2}$ and Christian R Voolstra ${ }^{1}$ \\ ${ }^{1}$ Red Sea Research Center, Division of Biological and Environmental Science and Engineering, King Abdullah \\ University of Science and Technology (KAUST), Thuwal, Saudi Arabia; ${ }^{2}$ Marine Chemistry and Geochemistry \\ Department, Woods Hole Oceanographic Institution, Woods Hole, MA, USA; ${ }^{3}$ Department of Ecology and \\ Evolutionary Biology, University of California, Los Angeles, CA, USA and ${ }^{4}$ Australian Institute of Marine \\ Science and College of Science and Engineering, James Cook University Townsville, Townsville, Queensland, \\ Australia
}

\begin{abstract}
Reef-building corals are well regarded not only for their obligate association with endosymbiotic algae, but also with prokaryotic symbionts, the specificity of which remains elusive. To identify the central microbial symbionts of corals, their specificity across species and conservation over geographic regions, we sequenced partial SSU ribosomal RNA genes of Bacteria and Archaea from the common corals Stylophora pistillata and Pocillopora verrucosa across 28 reefs within seven major geographical regions. We demonstrate that both corals harbor Endozoicomonas bacteria as their prevalent symbiont. Importantly, catalyzed reporter deposition-fluorescence in situ hybridization (CARD-FISH) with Endozoicomonas-specific probes confirmed their residence as large aggregations deep within coral tissues. Using fine-scale genotyping techniques and single-cell genomics, we demonstrate that $P$. verrucosa harbors the same Endozoicomonas, whereas $S$. pistillata associates with geographically distinct genotypes. This specificity may be shaped by the different reproductive strategies of the hosts, potentially uncovering a pattern of symbiont selection that is linked to life history. Spawning corals such as $\boldsymbol{P}$. verrucosa acquire prokaryotes from the environment. In contrast, brooding corals such as $S$. pistillata release symbiont-packed planula larvae, which may explain a strong regional signature in their microbiome. Our work contributes to the factors underlying microbiome specificity and adds detail to coral holobiont functioning.

The ISME Journal (2017) 11, 186-200; doi:10.1038/ismej.2016.95; published online 8 July 2016
\end{abstract}

\section{Introduction}

Coral reefs are declining globally at unsustainable rates (Descombes et al., 2015), driven by stressors including increasing sea surface temperatures, overfishing and anthropogenic inputs (De'ath et al., 2012). Devising strategies for mitigating future reef loss is challenging because corals are 'metaorganisms', comprised not only of the host itself, but also

Correspondence: A Apprill, Marine Chemistry and Geochemistry Department, Woods Hole Oceanographic Institution, Woods Hole, MA 02543, USA.

E-mail: aapprill@whoi.edu

or CR Voolstra, Red Sea Research Center, Division of Biological and Environmental Science and Engineering, King Abdullah University of Science and Technology (KAUST), Thuwal 239556900, Saudi Arabia.

E-mail: christian.voolstra@kaust.edu.sa

Received 9 January 2016; revised 19 April 2016; accepted 7 June 2016; published online 8 July 2016 symbiotic algae of the genus Symbiodinium, viruses, bacteria, archaea and fungi. This complex consortium is referred to as the coral holobiont (Rohwer et al., 2002; Knowlton and Rohwer, 2003). The beststudied members of the coral holobiont are the coral animal host and the Symbiodinium algae, which provide most of the host's energy requirements through photosynthates. In return, the symbiotic algae receive a safe refuge, consistent sunlight and nutrients (Goodson et al., 2001).

Although coral-prokaryotic associations have been recognized since the late 1970s (Ducklow and Mitchell, 1979; Williams et al., 1987), recent advancements in sequencing technology have revealed that microbes associated with corals are distinct from those in the seawater and are diverse, totaling several hundred species (Rohwer et al., 2001; Kellogg, 2004; Wegley et al., 2004; Sunagawa et al., 2009; Roder et al., 2013, 2014; Bayer et al., 2013b; Pantos et al., 2015). The high diversity of 
these microorganisms, with many belonging to uncultured genera, makes untangling the complicated interactions between microbes, the coral host and the algal dinoflagellate partners challenging. However, certain microbial species in the coral holobiont can fix nitrogen (Lesser et al., 2004; Lema et al., 2014), metabolize sulfur (Raina et al., 2009), provide antibiotic compounds (Reshef et al., 2006; Ritchie, 2006) and probably have roles in various other biogeochemical cycles (Kimes et al., 2010). Moreover, particular microbes appear to be 'core' symbionts of the holobiont, always associating with a specific coral host, and likely providing important functional benefits to the coral holobiont (Rohwer et al., 2001; Speck and Donachie, 2012; Jessen et al., 2013; Rodriguez-Lanetty et al., 2013; Bayer et al., 2013b; Ainsworth et al., 2015).

One of the potential 'core' symbionts of many corals are the Endozoicomonas, members of the Gammaproteobacteria's Oceanospirillaceae family (Morrow et al., 2012; Speck and Donachie, 2012; Apprill et al., 2013; Jessen et al., 2013; RodriguezLanetty et al., 2013; Bayer et al., 2013b; Lesser and Jarett, 2014; Morrow et al., 2014; Pantos et al., 2015). These bacteria also frequently associate with other marine organisms, including gorgonians (Correa et al., 2013; La Rivière et al., 2013; Vezzulli et al., 2013; Bayer et al., 2013a; Ransome et al., 2014), ascidians (Dishaw et al., 2014), tube worms (Forget and Juniper, 2013), mollusks (Jensen et al., 2010; Hyun et al., 2014) and fish (Mendoza et al., 2013). Although the sequence-based evidence for Endozoicomonas as a core microbiome member of certain corals is compelling, most studies have used corals from only one geographic location and often methods across studies are not standardized, making comparative analyses difficult. A comprehensive study of global reefs, with fine-scale attention to Endozoicomonas genotypes, is required to understand worldwide patterns of association between Endozoicomonas symbionts and corals.

Here, we examine the microbiomes of Stylophora pistillata and Pocillopora verrucosa across their global distribution to better understand the bacterial and archaeal community composition and governing principles, as well as the fine-scale specificity of their core symbionts. We predict that if microorganisms are important to healthy coral functioning and have co-evolved with corals, then coral microbiomes should be similar worldwide. Indeed, both coral species harbor Endozoicomonas as their abundant symbiont, which was found to reside in aggregates within coral tissues. Furthermore, S. pistillata displayed a highly geographically structured microbiome and was associated with Endozoicomonas genotypes unique to each geographic region. In contrast, $P$. verrucosa exhibited a weakly geographically structured microbiome and contained similar Endozoicomonas symbionts across large spatial scales. This suggests that microbial structure in corals may be linked to life history traits, and advances our understanding of coral holobiont acquisition and functioning.

\section{Materials and methods}

Site description and sampling

A total of 28 reefs were sampled in seven major geographical regions across the global range of the corals S. pistillata and $P$. verrucosa (Figure 1; Supplementary Table 1). A map of the sample sites was created in python using the package 'basemap', and the code to recreate the map is available at https://github.com/neavemj/microbiomeMap. At each reef, replicate colonies of each coral species were sampled (if present) using snorkel or SCUBA at depths between 2 and $10 \mathrm{~m}$. Colonies were sampled by removing $\sim 5 \mathrm{~cm}^{2}$ fragments using a hammer and chisel, which were then placed into Whirl-Pak bags (Nasco, Salida, CA, USA) underwater. At the surface, one fragment designated for DNA extraction was wrapped in aluminum foil and immediately frozen in liquid nitrogen, except for the Indonesian samples, which were preserved in 95\% ethanol. A second coral fragment was placed into a vial of $4 \%$ paraformaldehyde for microscopy analysis. Back at the laboratory, the DNA samples were frozen to $-80^{\circ} \mathrm{C}$ and the paraformaldehyde samples were stored at $4{ }^{\circ} \mathrm{C}$.

At all of the reefs except those in Indonesia, the physiochemical properties of the seawater were investigated. Temperature, salinity, dissolved oxygen and $\mathrm{pH}$ were measured using a YSI EXO1 MultiParameter Water Quality Sonde (Yellow Springs, $\mathrm{OH}$, USA), except at the Red Sea sites, which were measured using a WTW Multi 3500i multimeter (Weilheim, Germany). In addition, seawater surrounding the reefs was sampled for inorganic nutrients $\left(250 \mathrm{ml}\right.$, frozen to $-20^{\circ} \mathrm{C}$ ) and planktonic cell counts $(1 \mathrm{ml}$, fixed with $4 \%$ paraformaldehyde and frozen in liquid nitrogen) and analyzed as previously reported (Apprill and Rappé, 2011). For seawater microbial community analysis, several liters of seawater was collected, placed on ice and passed through $25 \mathrm{~mm}, 0.2 \mu \mathrm{m}$ filters (Millipore, Billerica, MA, USA) until saturated using a peristaltic pump. The seawater samples and filters were then stored at $-80^{\circ} \mathrm{C}$ until analysis.

\section{DNA extraction and host phylogeny}

Coral fragments were removed from the $-80^{\circ} \mathrm{C}$ freezer and immediately airbrushed using cold $1 \times$ phosphate-buffered saline (PBS). DNA was extracted from the airbrushed tissue and seawater filters with the PowerPlant Pro DNA isolation kit (MoBio Laboratories, Carlsbad, CA, USA) according to the manufacturer's instructions. S. pistillata host genotypes were assessed by amplifying the cytochrome oxidase I (COI) gene using the primer set LCO1: GGTCAACAAATCATAAAGATATTGG and HCO2: 

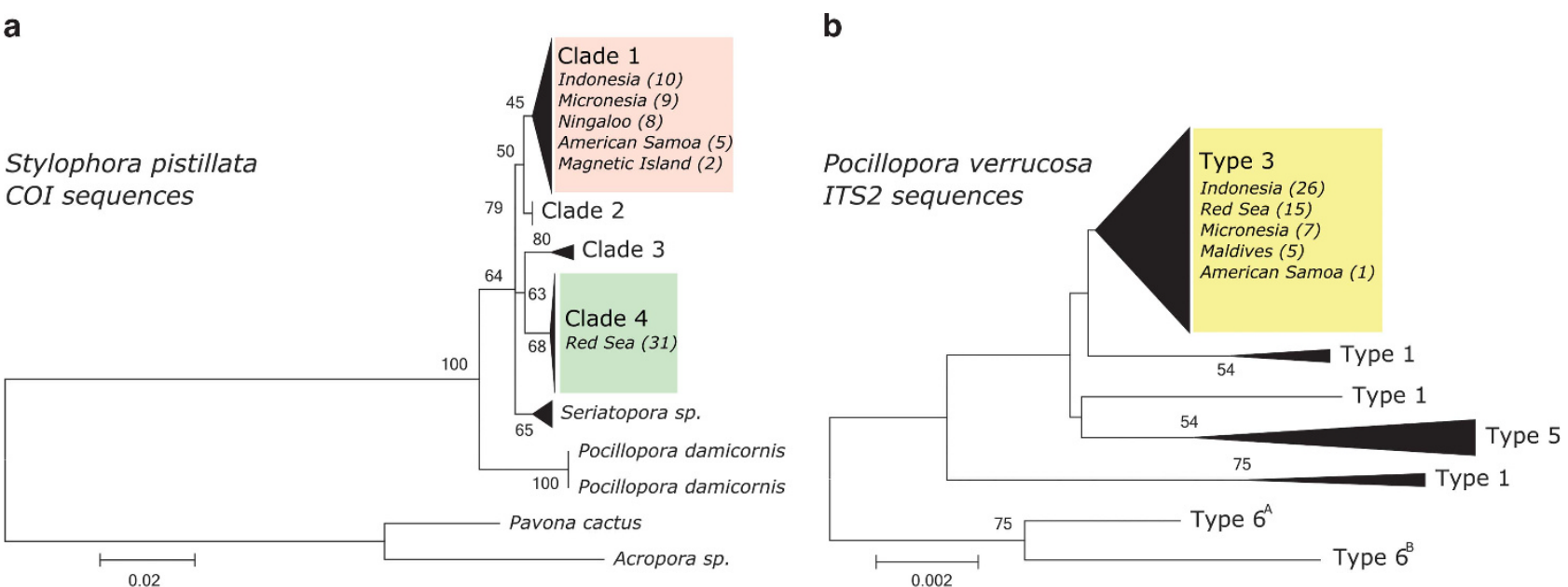

c
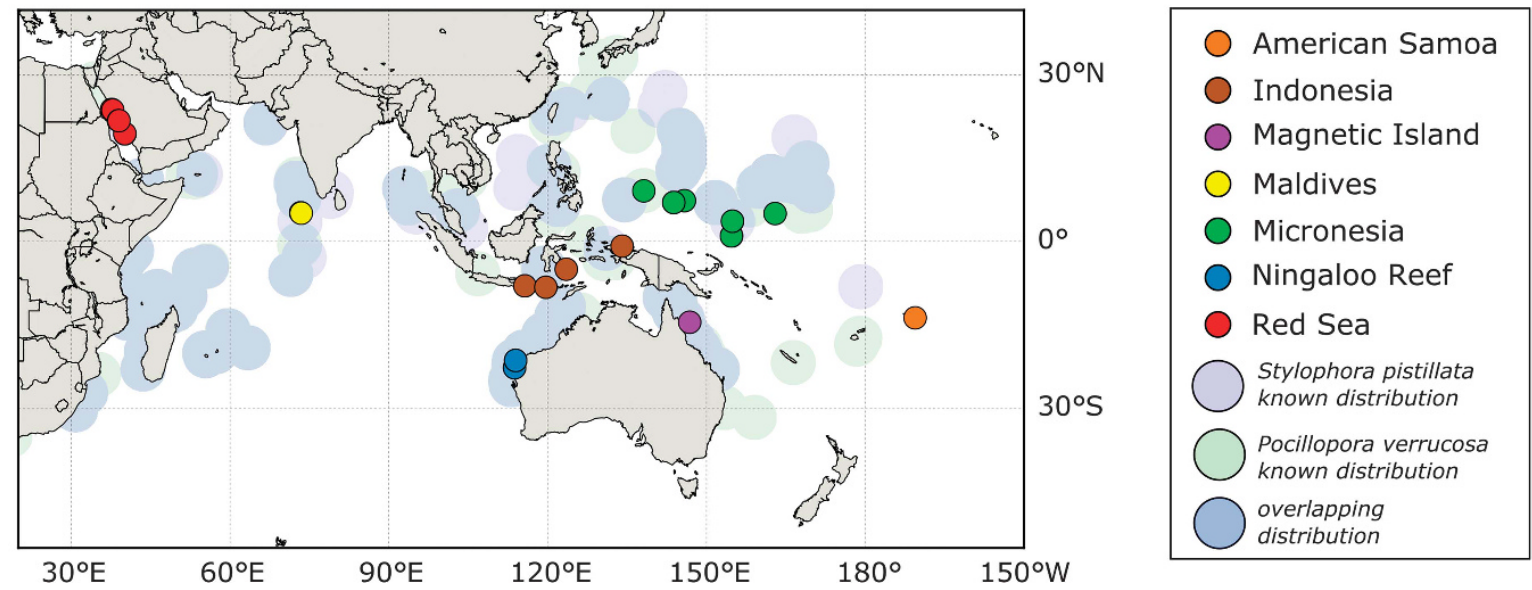

Figure 1 Coral host distribution and phylogenetic relationships of $S$. pistillata and $P$. verrucosa across global scales. Maximum Likelihood phylogenetic comparison of S. pistillata COI sequences with 'clades' from Keshavmurthy et al. (2013) (a), and Minimum Evolution comparison of $P$. verrucosa ITS2 sequences with 'types' from Pinzón et al. (2013) (b), and map of the reefs where corals were sampled in relation to their known distributions (c) obtained from the Ocean Biogeographic Information System (www.iobis.org).

TAAACTTCAGGGTGACCAAAAAATCA following Folmer et al. (1994). P. verrucosa host genotypes were determined using the internal transcribed spacer region 2 (ITS2) with the coral-specific primers ITSc2-5: AGCCAGCTGCGATAAGTAGTG and R28S1: GCTG CAATCCCAAACAACCC following Flot et al. (2008).

Amplified COI genes from the S. pistillata specimens were sequenced in the forward direction using an ABI3730xl genetic analyzer (GENEWIZ, South Plainfield, NJ, USA). Sequences were then trimmed for quality using CodonCode Aligner v3.7.1.2 (Centerville, MA, USA) and aligned with COI sequences previously reported (Keshavmurthy et al., 2013) using MUSCLE (Edgar, 2004). A Maximum Likelihood phylogenetic tree with 1000 bootstraps was created from the alignment using MEGA 6 (Tamura et al., 2013). The ITS2 genes from $P$. verrucosa specimens were sequenced in both the forward and reverse direction using an ABI3730xl genetic analyzer (GENEWIZ). The forward and reverse sequences were both required because more than one haplotype was often seen per individual and both sequences were needed to deconstruct the chromatograph into individual alleles (Champuru v1.0 (Flot, 2007)). Deconstructed alleles were then aligned with sequences from Pinzón et al. (2013) using MUSCLE (Edgar, 2004) and a Minimum Evolution phylogenetic tree with 1000 bootstraps was created using MEGA 6 (Tamura et al., 2013). All sequences were deposited in GenBank (COI accession numbers KR105650KR105714, ITS accession numbers KR105715KR105768).

Single-cell SSU rRNA gene sequencing was conducted on cells isolated from Red Sea corals collected from Al Fahal in February 2013 (Supplementary Table 1). Briefly, fragments of $S$. pistillata colonies were collected as previously described, placed on ice and immediately taken to the laboratory where they were airbrushed with cold PBSE ( $1 \times$ PBS, $10 \mathrm{~mm}$ EDTA). The slurry was then gently spun down, resuspended in glyTE $(10 \times \mathrm{TE}$, $50 \%$ glycerol) and frozen to $-80^{\circ} \mathrm{C}$. The cells were shipped on dry ice to the Bigelow Single Cell 
Genomics Center (East Boothbay, ME, USA), where they were sorted using fluorescence-activated cell sorting (FACS). The DNA was then extracted and amplified using multiple displacement amplification (MDA), and the full-length SSU rRNA gene from single cells was sequenced (GenBank accession numbers KR233695-KR233752) as described by Stepanauskas and Sieracki (2007).

\section{Microbiome sequencing and analysis}

Bacterial and Archaeal V4 SSU rRNA gene fragments were amplified from the coral and seawater DNA using the primer set 515F: GTGCCAGCMGCCG CGGTAA and 806RB: GGACTACNVGGGTWTC TAAT (Caporaso et al., 2012; Apprill et al., 2015) combined with an Illumina (San Diego, CA, USA) adapter, $8 \mathrm{bp}$ index sequence, $10 \mathrm{bp}$ pad sequence and $2 \mathrm{bp}$ linker sequence in order to use the dualindex sequencing strategy outlined by Kozich et al. (2013). The V4 SSU rRNA binding site for the reverse primer was modified as discussed by Apprill et al. (2015) to more efficiently capture bacteria from the SAR11 clade. We did not utilize the $515 \mathrm{~F}$ primer optimized for Thaumarchaeota (Parada et al., 2015); however, using a metagenomics approach in $S$. pistillata and $P$. verrucosa, we found that Archaea were far less abundant than Bacteria (unpublished). The PCR amplicons were sequenced over two 250-bp paired-end Illumina MiSeq runs. To check for bias across the two runs, 11 of the samples were identically prepared and sequenced on both.

De-noising and other quality filtering procedures were completed in mothur v1.34.4 (Schloss et al., 2009). The make.contigs command was used to combine the reads, screen.seqs was used to remove sequences if they contained ambiguous positions or were longer than $255 \mathrm{bp}$, classify.seqs was used with the 'knn' method to classify the contigs against the SILVA database v119 (Quast et al., 2013), and the remove.lineage command removed contigs classified as chloroplast, mitochondria, eukaryota or were unknown. Finally, chimeras were removed using UCHIME (Edgar et al., 2011) with abundant sequences as the reference. The error rate of the sequencing was determined using seq.error and mock community samples, and was found to be $0.19 \%$. The sequenced negative controls returned few reads that were removed in the cleaning and filtering steps. The sequences were then subsampled to 7974, which captured most of the diversity and kept most samples. Raw sequences were deposited in the NCBI Sequence Read Archive (SRA) under BioProject number PRJNA280923.

Two different approaches were applied for sorting the cleaned sequences into taxonomic units: pairwise similarity (1\% and 3\%) and Minimum Entropy Decomposition (Eren et al., 2014) (MED). The MED approach uses Shannon entropy to identify nucleotide positions in a sequence alignment that are useful for discriminating operational taxonomic units (OTUs) and was able to discern finer scale differences in sequences compared with pairwise similarity approaches. For example, using 3\% OTU clustering, the most abundant overall OTU was present in both Micronesian and Red Sea S. pistillata, the MED technique, however, decomposed this sequence into OTUs that were specific to each geographic region (Figure 2; bottom boxplots). A custom python script was used to convert the cleaned fasta and group files from mothur into a MED-compatible format (available at: https:/github.com/neavemj/globalCoral Microbiomes/ 'headersMED.py'). MED was then run on the sequences using default settings and a minimum substantive abundance criterion $(M)$ of 158 (1578 852 sequences/10 000) as recommended (Eren et al., 2014). For comparative purposes, the same sequences were used to make $1 \%$ and $3 \%$ OTUs in mothur (Schloss et al., 2009). The sequences were aligned to the SILVA database v119 (Quast et al., 2013) using align.seqs, then pre. cluster was used with a difference of $2 \mathrm{bps}$ to reduce the dataset size. The sequences were re-classified using the SILVA database v119 to use cluster.split with the taxonomy option and a taxlevel of 5 to cluster the sequences. Following the clustering, 1\% and $3 \%$ OTU tables were created using make.shared. To ensure accurate comparisons, the remove.rare command was used to remove OTUs with an abundance lower than 158, matching the minimum abundance of MED OTUs above. The representative MED OTUs and $1 \%$ and 3\% OTU sequences were classified in mothur (Schloss et al., 2009) using the 'knn' method and the SILVA database v119. Analysis of molecular variance was used to look for significant differences in the microbiomes across sites using a Bray-Curtis similarity matrix and amova in mothur (Schloss et al., 2009).

All remaining statistical and graphical analyses were completed in $\mathrm{R}$ ( $\mathrm{R}$ Development Core Team, 2010). In the interest of reproducibility, an $\mathrm{R}$ markdown document containing complete commands for the analysis is available here: https:// github.com/neavemj/globalCoralMicrobiomes/ 'coralMicrobiomes.Rmd'. Briefly, after the OTU matrices and metadata were imported into $\mathrm{R}$, alpha diversity measures were calculated using $3 \%$ pairwise OTUs with the command plot_richness in the package 'phyloseq' (McMurdie and Holmes, 2013), and the measures were plotted as a boxplot in 'ggplot' (Wickham, 2009). To check for significant differences between the alpha diversity measures, the $\mathrm{R}$ base package 'stats' was used to perform a Kruskal-Wallis test and post hoc pairwise comparisons were conducted using a Dunn's test (Dinno, 2015) with Bonferroni corrections for multiple testing. Phyloseq was used to create barcharts with plot_bar and ordinations with plot_ordination using square-root transformed Bray-Curtis similarity matrices on MED OTUs (McMurdie and Holmes, 2013). Physiochemical data were correlated with the 

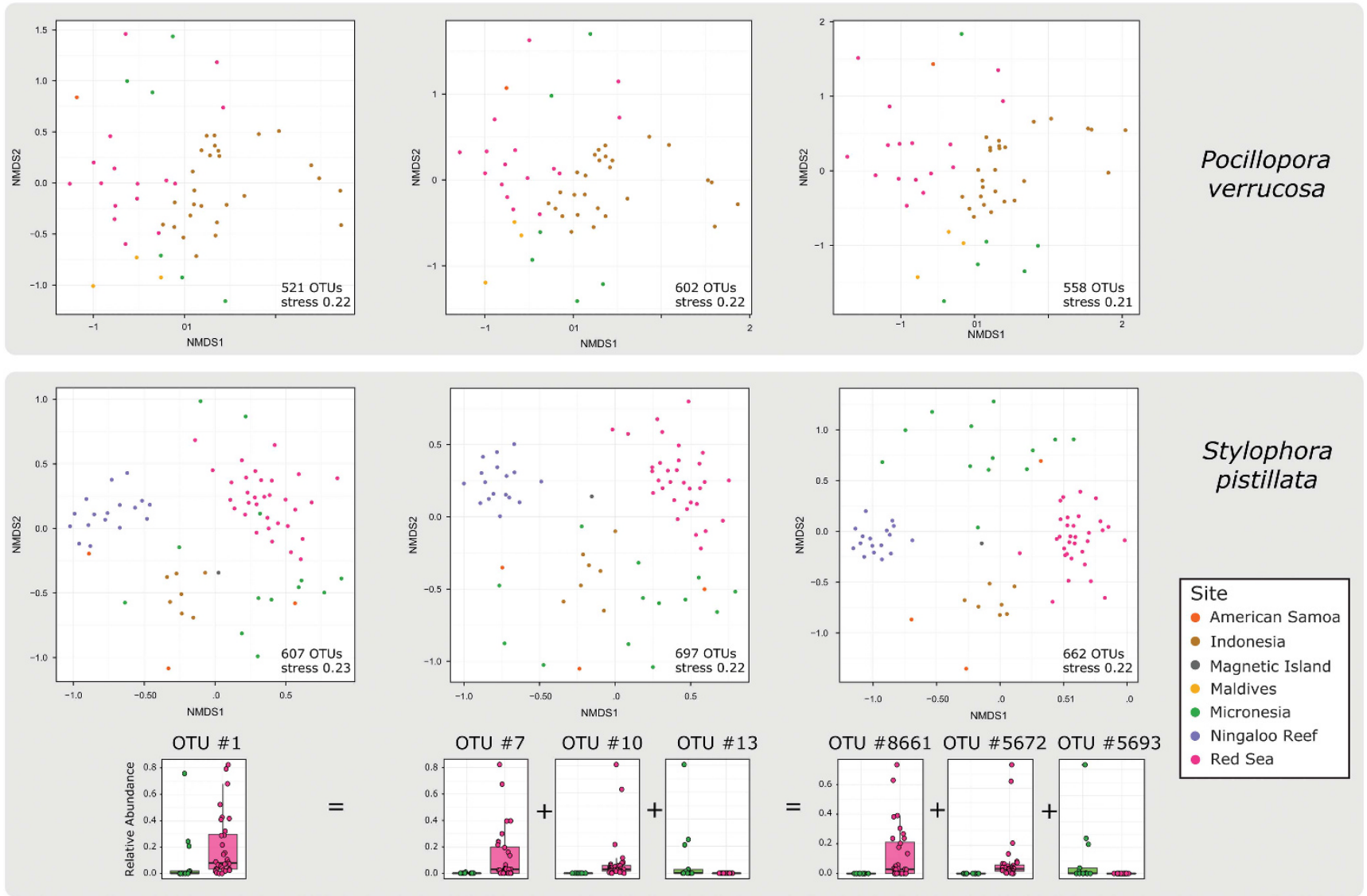

Figure 2 Clustering of coral specimens based on microbiome composition. Non-metric multidimensional scaling comparisons of $S$. pistillata and $P$. verrucosa microbiomes, comparing pairwise OTU clustering with the MED OTU approach to clustering SSU rRNA gene sequences (Eren et al., 2014). The bottom boxplots show how sequences that formed the most abundant OTU with $3 \%$ clustering (left) are divided into 1\% OTUs (middle) and MED OTUs (right), for two sample sites (Micronesia and Red Sea). This indicates that 3\% clustering detects no difference between the most abundant OTU within Micronesian and Red Sea corals, whereas 1\% OTUs and MED OTUs clearly show site-specificity.

microbiome ordinations using envfit in the $\mathrm{R}$ package 'vegan' and 999 permutations to test for significance (Oksanen et al., 2013), and SIMPROF analysis was run with 999 permutations using the R package ‘clustsig' (Whitaker and Christman, 2014).

\section{Endozoicomonas phylogeny}

Endozoicomonas bacteria were found to be the most prevalent symbionts associated with both corals and were analyzed further. The MED OTUs that classified to Endozoicomonas were aligned using the SINA alignment service (www.arb-silva.de/aligner/) (Pruesse et al., 2012), and imported into ARB (Ludwig et al., 2004). After manual refinement of the alignment, including exclusion of partially represented regions, a Maximum Likelihood tree containing the OTUs, plus Endozoicomonas sequences from several previous studies, was constructed. The tree was imported into $\mathrm{R}$ and metadata was added to the tree tips using plot_tree in phyloseq (McMurdie and Holmes, 2013). A heatmap of Endozoicomonas OTUs was also created using phyloseq and the plot_heatmap command. To check for significant differences in Endozoicomonas abundance across sites, negative binomial modeling was conducted using DESeq2 (Love et al., 2014; McMurdie and Holmes, 2014).

Catalyzed reporter deposition-fluorescence in situ hybridization (CARD-FISH)

Coral fragments that were preserved for microscopy analysis were removed from the $4 \%$ paraformaldehyde and rinsed three times in phosphate-buffered saline (PBS) for $20 \mathrm{~min}$ each. Samples were decalcified at $4{ }^{\circ} \mathrm{C}$ in $20 \%(\mathrm{w} / \mathrm{v})$ tri-sodium EDTA (pH 7-8) in distilled water changed daily for up to 10 days. The tissues were then dehydrated for 20 min each in $70 \%, 90 \%$ and $95 \%$ and two times in $100 \%$ ethanol, followed by three 20-min washes in HistoClear (National Diagnostics, Atlanta, GA, USA). The tissues were then embedded in paraffin and sectioned to 5 or $10 \mu \mathrm{M}$ using an 820 Rotary Microtome (American Optical, Buffalo, NY, USA). The sections were deparaffinated by heating to $60^{\circ} \mathrm{C}$ and washing 
for $10 \mathrm{~min}$ in HistoClear three times, then 10-min washes of $95 \%, 80 \%$ and $70 \%$ ethanol. The sections were permeabilized in $0.2 \mathrm{M} \mathrm{HCl}$ for $12 \mathrm{~min}, 20 \mathrm{~mm}$ Tris/HCL for $10 \mathrm{~min}, 0.5 \mu \mathrm{g} \mathrm{ml}^{-1}$ proteinase $\mathrm{K}$ for $5 \mathrm{~min}$ at $37^{\circ} \mathrm{C}$ and a final rinse of $20 \mathrm{~mm}$ Tris/HCL for $10 \mathrm{~min}$. Next, sections were covered in hybridization buffer (35\% formamide, $0.9 \mathrm{M} \mathrm{NaCl}, 20 \mathrm{~mm}$ Tris/HCL (pH 7.4), 0.01\% SDS, 1\% blocking reagent (Roche, Basel, Switzerland), 10\% (w/v) dextran sulfate) and placed in a humidity chamber for $20 \mathrm{~min}$ at $35^{\circ} \mathrm{C}$. The tissues were then hybridized for $3 \mathrm{~h}$ with horseradish peroxidase-labeled $0.5 \mathrm{ng}^{-1}$ Endozoi663: 5'-GGAAATTCCACACTCCTC-3' (Bayer et al., 2013b) or horseradish peroxidase-labeled EUB338: 5'-GCWGCCWCCCGTAGGWGT-3' (Daims et al., 1999) in hybridization buffer at $35^{\circ} \mathrm{C}$. In addition, control samples were hybridized with horseradish peroxidase-labeled NON338 probe: 5'-ACTCCTACGGGAGGCAGC-3' (Amann et al., 1990), as well as no probe, under the same conditions. Following hybridization, the slides were washed two times for $15 \mathrm{~min}$ at $35^{\circ} \mathrm{C}$ in fresh hybridization wash $(80 \mathrm{~mm} \mathrm{NaCl}, 20 \mathrm{~mm}$ Tris/HCl (pH 8.0), 5 mM EDTA (pH 8.0), 0.01\% SDS), then for $15 \mathrm{~min}$ in $1 \times \mathrm{PBS}$ at room temperature. For CARD amplification, a fresh solution of $0.15 \% \mathrm{H}_{2} \mathrm{O}_{2}$ in $1 \times$ PBS was mixed in a ratio of 1:100 with amplification buffer $(1 \times$ PBS, $0.1 \%$ blocking reagent (Roche), $2 \mathrm{~m} \mathrm{NaCl,} 10 \%$ (w/v) dextran sulfate). Cy3-labeled tyramide $\left(1 \mathrm{mg} \mathrm{ml}^{-1}\right)$ was then added to the mix at a ratio of 1:500, applied to the slides and incubated for $20 \mathrm{~min}$ at $37^{\circ} \mathrm{C}$ in a dark humidity chamber. Following the amplification, the slides were washed in $1 \times$ PBS at room temperature for $15 \mathrm{~min}$, then for $1 \mathrm{~min}$ in distilled water. To reduce background interference from the naturally autofluorescent coral tissues, the slides were covered with $6 \% \mathrm{H}_{2} \mathrm{O}_{2}$ in ethanol overnight (14-16 h) according to Fukatsu et al. (2007) and mounted using the Vectashield DAPI mounting strain (Vector Laboratories, Burlingame, CA, USA).

Samples were imaged using a $\times 63$ objective under $\times 1.6$ optovar on a Zeiss Axio Observer.Z1 fluorescent microscope with an AxioCam MRm camera using the Zen Pro 2011 software (Carl Zeiss Microscopy, Thornwoods, NY, USA) and Cy3, DAPI and PA-GFP emission detected at 570, 395 and $495 \mathrm{~nm}$, respectively. For each colony (eight S. pistillata from Ningaloo and Micronesia, three $P$. verrucosa from Maldives and Micronesia), 10-41 sections were examined with the Endozoicomonas probe, and compared with control specimens (no probe, nonsense probe) of selected adjacent sections of the same samples. Hematoxylin and eosin staining (ClearView, BBC Biochemical, Mount Vernon WA, USA) was conducted on sections adjacent to those that were positive for Endozoicomonas, and sections were imaged under $\times 4$ and $\times 60$ objectives using an EVOS FL Cell Imaging System (Life Technologies, Carlsbad, CA, USA).

\section{Results}

\section{Coral host phylogeny}

To ensure valid comparisons of coral species across distant geographic regions, host genotype was resolved using the cytochrome oxidase $I$ (COI) gene for $S$. pistillata and the internal transcribed spacer region (ITS2) for $P$. verrucosa (Figure 1) (Flot et al., 2008; Keshavmurthy et al., 2013). The rapidly evolving ITS2 region was used to identify $P$. verrucosa because many Pocilloporid corals cannot be differentiated using the slower evolving COI gene, including $P$. damicornis and $P$. verrucosa, two closely related species that were sympatric within our study range (discussed by Pinzón et al. (2013) and Flot et al. (2008)). Species of Stylophora, on the other hand, can be differentiated using the COI gene (see Keshavmurthy et al. (2013)), eliminating the need to sequence the ITS2 region for our Stylophora specimens. All $S$. pistillata colonies formed a monophyletic clade, except for those from the Red Sea, which formed a sister branch (Figure 1a). These results correspond to clade 1 and clade 4 from Keshavmurthy et al. (2013) and suggest that Red Sea $S$. pistillata are genetically isolated from the Indo-Pacific populations. The taxonomy of $P$. verrucosa was more complicated owing to higher diversity across the ITS2 gene and the presence of heterozygous individuals. Nevertheless, most of the samples we identified in the field as $P$. verrucosa could be assigned to Pocillopora 'type 3' (which includes P. verrucosa) sensu Pinzón et al. (2013) (Figure 1b). Pocillopora colonies that did not cluster with the 'type 3' samples were not used in subsequent microbiome analyses. Hereafter, the 'type 3' Pocillopora will be referred to as Pocillopora verrucosa. This investigation of host genotypes provided some confidence that the global coral samples could be examined in a comparative framework. It should be noted, however, that coral phylogeny is a frequently changing field and that host subspecies cannot be ruled out.

\section{Fine-scale SSU rRNA diversity analysis}

High-throughput sequencing of hypervariable V4 region SSU rRNA gene amplicons (Sogin et al., 2006; Caporaso et al., 2012) was used to examine the bacterial and archaeal communities in corals (73 colonies $S$. pistillata, 53 colonies $P$. verrucosa) and the seawater surrounding those colonies (Supplementary Table 1). We examined two different approaches for categorizing the microbial sequences into OTUs for fine-scale specificity characterizations across global scales: pairwise similarity (both at $1 \%$ and 3\% cutoffs) and MED (Eren et al., 2014) (Figure 2). We found that MED resolved finer clusters than the pairwise similarity approaches, particularly when compared with $3 \%$ pairwise similarity (Figure 2). In addition, we conducted single-cell sorting of microbial cells and subsequent full-length 
SSU rRNA gene Sanger sequencing for S. pistillata acquired from the Red Sea. To date, few studies have used single-cell genomics for multicellular animals and several challenges were encountered, largely arising owing to the complex consortium of cells within the coral holobiont, such as coral mitochondria, viruses, fungi, Symbiodinium and naturally fluorescent coral cells that interfere with the sorting process. Nevertheless, through careful selection of bacteria-like size ranges during the fluorescence- activated cell sorting, we recovered 67 bacterial cells from a 384-well plate, 66 of which were identified as Endozoicomonas. Convincingly, the abundant Red Sea MED OTUs were identical to the SSU rRNA gene sequences obtained from single-cell sequencing (Figure 3, single-cell types A and B), suggesting that MED OTUs obtained from high-throughput sequencing indeed represent biologically relevant units. For these reasons, we used MED OTUs for further global comparisons, except for the calculation of alpha

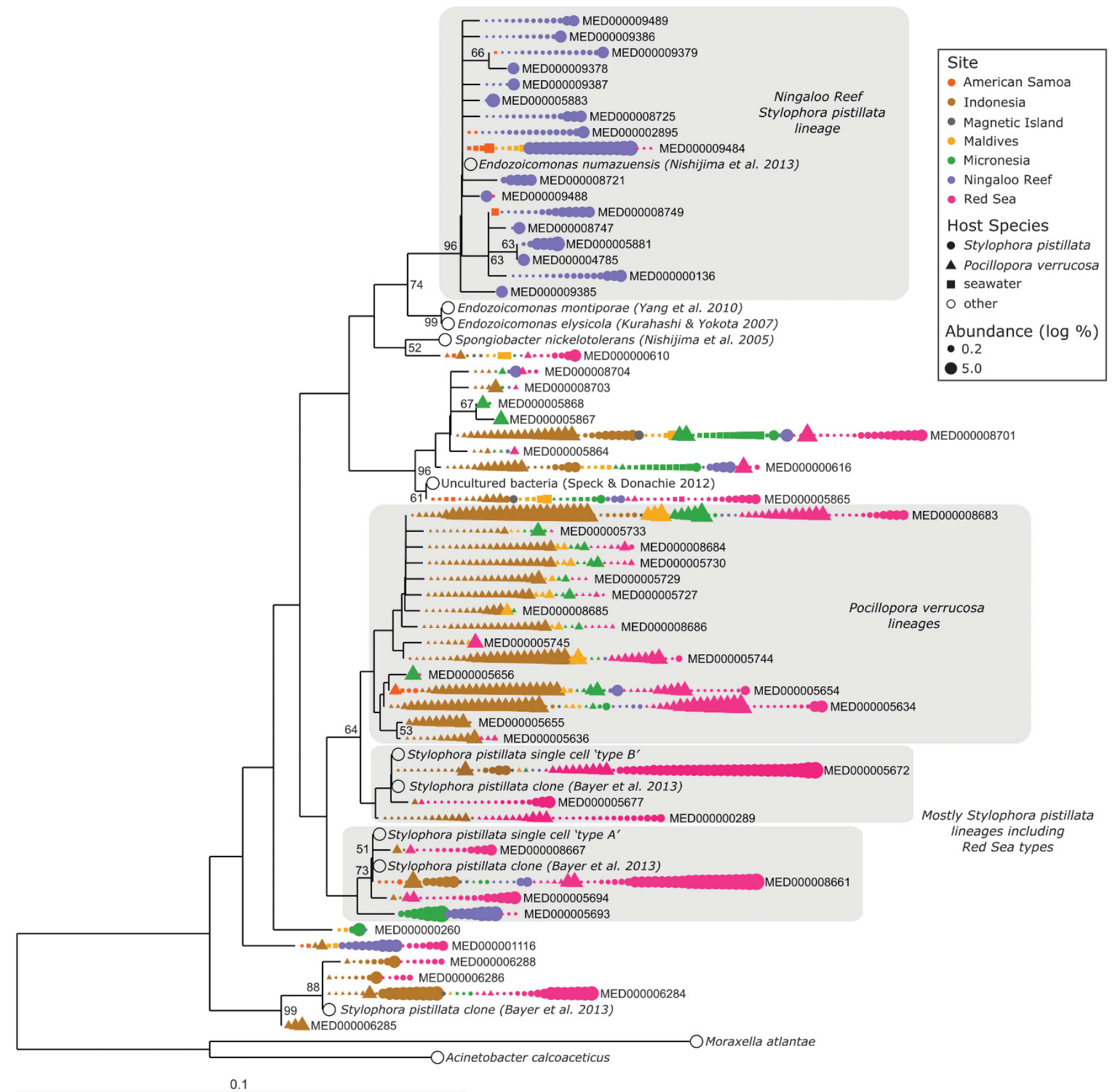

Figure 3 Phylogeny of coral-associated Endozoicomonas symbionts. Neighbor-joining tree containing all Endozoicomonas MED OTUs detected in the study, overlaid with the host species and geographic location of sequences clustered within the OTUs. Sequences detected in seawater are also represented, as well as the V4 region from full-length Endozoicomonas SSU rRNA gene sequences from the single-cell sorts and other coral studies. The scale bar corresponds to the estimated number of nucleotide changes per sequence position. 
diversity measures, which are not yet implemented for MED methodology.

Corals are highly selective for their microbiome compared with seawater

Comparisons of Chao1 and Simpson diversity indices between the coral and seawater microbial communities revealed that, on a global scale, corals harbored a more selective community of microorganisms compared with those in the surrounding seawater, as evidenced by significantly lower richness and evenness compared with the seawater communities (Supplementary Figure 1). This trend was consistent among the reefs, despite some variation in environmental parameters, such as the concentrations of inorganic nutrients $(0.04-2.0 \mu \mathrm{M}$ nitrate+nitrite) and temperature $\left(24.7-30.5^{\circ} \mathrm{C}\right)$, as well as among concentrations of picoeukaryotes (341-23 809 cells per milliliter) in the waters surrounding the corals (Supplementary Tables 2 and 3 ). For $S$. pistillata and $P$. verrucosa, alpha diversity of the associated microbes was not significantly different (Supplementary Figure 1), and the Chao1 richness metric predicted an average of 217 (min 26, max 851) coral-associated microbial taxa using 3\% OTU clustering.

Different geographic structuring of the microbiome in $S$. pistillata and $P$. verrucosa, yet selection for Endozoicomonas bacteria

Multivariate analysis of the SSU rRNA gene MED OTUs revealed that the microbiomes of $S$. pistillata and $P$. verrucosa partitioned differently according to geographic location (Figure 2). Specifically, $S$. pistillata microbiomes were strongly geographically segregated, but this structuring was weaker for $P$. verrucosa. Analyses of molecular variance (AMOVA) demonstrated that the $S$. pistillata microbiomes were significantly distinct from each other at four of the six major geographic locations, including Indonesia, Micronesia, Ningaloo Reef and the Red Sea (Supplementary Table 4). In contrast, the $P$. verrucosa microbiomes did not show a clear segregation according to geographic location (Figure 2), and the only significantly different corals were from the Red Sea (Supplementary Table 4). These patterns were also seen in a Similarity Profile Analysis (SIMPROF), which clustered samples without any a priori assumptions regarding sample origin (Supplementary Figure 2).

To test for significant environmental correlations with microbiome composition, the environmental variables were fitted to the microbiome ordinations (Supplementary Figure 3). The Red Sea sites had higher salinity, Ningaloo Reef sites had higher silicate concentrations, and the Micronesian sites displayed higher temperatures compared with the other sites, which correlated with the microbiome composition. Although these correlations are difficult to interpret in a cause-and-effect framework, it may suggest that environmental factors had some influence on the coral microbiomes. However, this influence is unlikely to account for the different microbiome specificity seen between $S$. pistillata and $P$. verrucosa, because the corals were collected at the same reefs at the same time and presumably experienced the same physiochemical conditions.

Taxonomically, the microbiomes of both $S$. pistillata and $P$. verrucosa were dominated by bacteria belonging to the Gammaproteobacteria and Alphaproteobacteria (Supplementary Figures 4 and 5). Higher variability between the corals was seen at the genus level, although Endozoicomonas was the most prevalent taxa for both corals. Endozoicomonas OTUs (relative abundance $>1 \%$ ) were detected in $79 \%$ of $S$. pistillata samples and $85 \%$ of $P$. verrucosa samples across their global distribution, suggesting that these bacteria are prevalent microbiome members. Along with the widespread Endozoicomonas associations, the corals also contained a diverse community of prokaryotic groups (Supplementary Figures 4 and 5). For example, the $S$. pistillata samples from Ningaloo Reef contained unusually large abundances of Pseudomonas (average 32\%, $\min 10 \%$, max $68 \%$ ), and P. verrucosa from several Indonesian sites contained substantial amounts of Prochlorococcus (average 15\%, min 9\%, max 21\%). Although some of these prokaryotic associates may have important roles in the coral holobiont, here, we focused on the Endozoicomonas owing to their consistent prevalence and the opportunity to assess host specificity for Endozoicomonas across global scales. The coral-associated microbes were different to those in the seawater, which was dominated by members of the Cyanobacteria and SAR11 clade of Alphaproteobacteria (Supplementary Figure 6). Archaea were generally rare in both corals and seawater, although members of the Euryarchaeota occasionally accounted for up to $25 \%$ of the seawater microbial community (Supplementary Figure 6).

Intimate association of Endozoicomonas bacteria with their coral hosts

The spatial location of the Endozoicomonas endosymbionts within coral tissues was examined using CARD-FISH (Figure 4). Microscopic CARD-FISH images revealed that Endozoicomonas symbionts were located deep within coral tissues, confirming an important and intimate association with their hosts (Figure 4) (Bayer et al., 2013b). In both coral species, the Endozoicomonas cells were arranged in cyst-like, dense cellular aggregations, spanning $\sim 50 \mu \mathrm{M}$ in diameter, which were confirmed with hematoxylin and eosin staining (Supplementary Figures 7 and 8). In S. pistillata, the Endozoicomonas cyst-like aggregations were intratentacular, bordering the epidermis and gastrodermis (Figure 4, Supplementary Figure 7), whereas in $P$. verrucosa, they were predominantly located within gastrodermal 

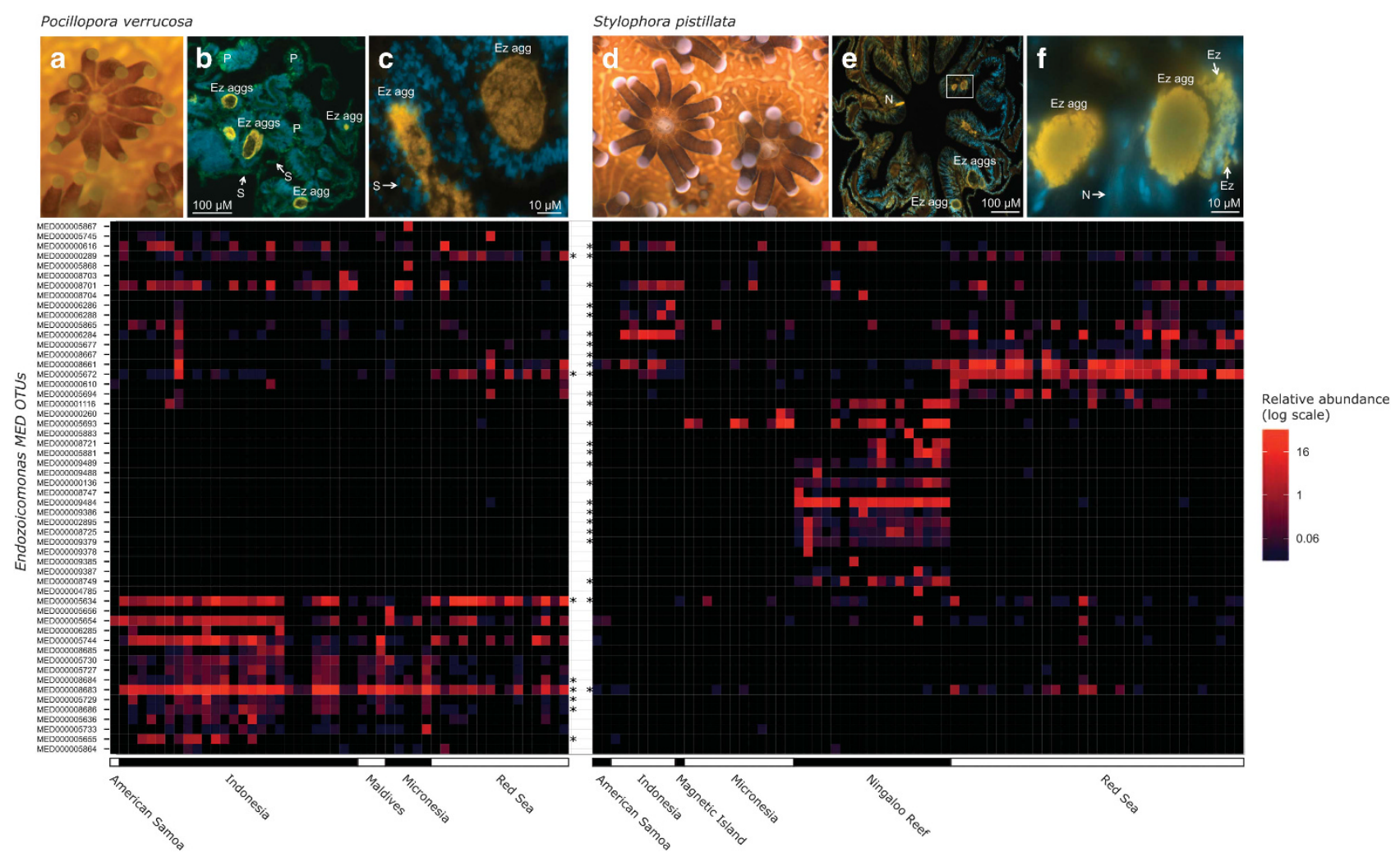

Figure 4 Light microscopy and FISH imaging of Endozoicomonas within the gastrodermal tissues of $P$. verrucosa (a-c) and within the tentacles surrounding the oral disc of $S$. pistillata $(\mathbf{d}-\mathbf{f})$, and a heatmap of Endozoicomonas OTU abundance and diversity in P. verrucosa and S. pistillata (bottom panel). Endozoicomonas cells are hybridized with the horseradish peroxidase-labeled probe targeting the Endozoicomonas bacteria and appear yellow. The blue staining is a general nucleic acid stain (DAPI) and (b) is additionally imaged under fluorescein isothiocyanate excitation and the green coloration is coral and Symbiodinium autofluorescence. Select Endozoicomonas cells (Ez) and cell aggregations (Ez agg), nematocysts (N), Symbiodinium cells (S) and coral pigment cells (P) are denoted. (f) is an inset of (e), and (c) is a representative magnified image from P. verrucosa. For the FISH images, non-cellular yellow coloration is present owing to coral tissue autoflurescence as well as probe sticking to nematocysts $(\mathrm{N})$. The bottom panel is a heatmap of the abundance (log-scale base 4) of Endozoicomonas MED OTUs detected in P. verrucosa and S. pistillata. Asterisks indicate Endozoicomonas OTUs that had significantly different abundances $(P<0.05)$ across the sites based on negative binomial modeling in DESeq2.

tissues and absent from the tentacles (Figure 4, Supplementary Figure 8).

Fine-scale coral-Endozoicomonas specificity across species and globally distant reefs

Almost all of the corals examined contained a dominant Endozoicomonas MED OTU (>75\%), and most also contained several less abundant types (Figure 4). S. pistillata and P. verrucosa were associated with host-specific Endozoicomonas MED OTUs, and few OTUs were present in both coral hosts. Moreover, the Endozoicomonas in $P$. verrucosa showed only slight geographic partitioning, whereas Endozoicomonas in S. pistillata were strongly grouped by geographic location, with 8 and 25 OTUs, respectively, demonstrating significantly different geographic abundances $(P<0.05$, Figure 4$)$, reflecting the total community results (Figure 2).

Phylogenetic analysis of the Endozoicomonas OTUs revealed site- and host-specificity patterns within this bacterial genus (Figure 3). As observed in Figure 4, Endozoicomonas associated with $S$. pistillata were generally distinct OTUs at the geographically distant reefs. Particularly distinct were Endozoicomonas OTUs from Ningaloo Reef of Australia, which formed a monophyletic branch and the only other geographic location represented here were from sequences recovered from seawater (Figure 3). Interestingly, Endozoicomonas from the other geographic locations did not always belong to the same genetic lineage. For example, an abundant Red Sea OTU (MED000005672) was more closely related to P. verrucosa's Endozoicomonas from the Red Sea and Indonesia, than to the other abundant S. pistillata Red Sea OTUs (MED000008661 and MED000006284). Several other Endozoicomonas types within Indonesia, Micronesia and Ningaloo Reef corals belonged to disparate lineages, suggesting that a number of genetically distinct Endozoicomonas OTUs coexist in these corals. In contrast, most Endozoicomonas taxa associated with 
$P$. verrucosa belonged to the same lineage and were shared across geographic areas (Figure 3).

\section{Discussion}

Different microbiome structuring for S. pistillata and

P. verrucosa

$S$. pistillata and $P$. verrucosa are closely related coral species both belonging to the family Pocilloporidae. They probably diverged relatively recently, near the beginning of the Neogene period around 23 million years ago (Park et al., 2012). Despite this phylogenetic similarity, we found significant differences in the microbiomes of the two corals and in the apparent forces structuring the microbiome. Specifically, S. pistillata microbiomes were strongly clustered according to geographic location, whereas in $P$. verrucosa, the groupings were much weaker. These differences may have arisen because of the distinctive reproductive strategies of the corals; S. pistillata is a brooder (Hall and Hughes, 1996; Shlesinger et al., 1998) and $P$. verrucosa is a broadcast spawner (Pinzón et al., 2013). In fact, some authors have suggested that the divergence between Stylophora and Pocillopora was established by the evolution of these different reproductive modes (Schmidt-Roach et al., 2014). Previous studies of the broadcast spawning coral, $P$. meandrina, found that larvae less than 3 days old were sterile of prokaryotes (Apprill et al., 2009) and some of their microbiome originates from the surrounding seawater (Apprill et al., 2012). The gametes of other broadcast spawning corals are also sterile of prokaryotic cells, which are acquired later from the water column (Sharp et al., 2010; Ceh et al., 2013). In contrast, the brooding coral, Porites astreoides, passed microbes vertically from parent to offspring (Sharp et al., 2012). Assuming these patterns hold true for the corals studied here, it could explain the observed geographic structuring patterns. Namely, the brooding coral $S$. pistillata may vertically transfer microbes to their offspring, thereby tightly controlling the microbiome and its evolution, and resulting in strong geographic structuring. Conversely, if $P$. verrucosa larvae were sterile and their microbial associates were acquired from seawater, symbiont mixing between distinct reefs is likely, resulting in weaker geographic structuring. Moreover, Pocillopora species have unusually large areas of genetic connectivity and low species diversity compared with other cosmopolitan coral species, such as S. pistillata (Pinzón et al., 2013; Robitzch et al., 2015). This potentially high gene flow between Pocillopora species is probably facilitated by exceptionally long planktonic stages. For example, P. damicornis, which is closely related to $P$. verrucosa, has a planktonic stage of more than 100 days in aquaria (Richmond, 1987), which would allow mixing of populations from relatively distant oceanic basins. High connectivity between hosts would almost certainly provide opportunities for symbiont sharing, potentially explaining the weaker geographic signature in $P$. verrucosa microbiomes.

The geographic structuring pattern for the overall microbial communities was even more pronounced for Endozoicomonas, the most abundant symbiont in both corals. For $S$. pistillata, each large geographic region contained unique Endozoicomonas OTUs. This was not seen in $P$. verrucosa, which often harbored the same Endozoicomonas OTUs across large geographic ranges. Reproductive differences in the corals may again account for this pattern. If Endozoicomonas are passed vertically in the brooder S. pistillata, this could produce strong regional clustering, whereas if Endozoicomonas are acquired from the seawater in $P$. verrucosa, the endosymbiont types may be shared. The higher genetic connectivity of Pocillopora (Pinzón et al., 2013; Robitzch et al., 2015) would also allow for greater sharing of Endozoicomonas genotypes. Interestingly, Hester et al. (2015) studied three spawning coral species (Acropora rosaria, A. hyacinthus and Porites lutea) and did not find stable symbionts that were universally associated with one host, similar to $P$. verrucosa here. Our study was able to extend this analysis by including the brooder, $S$. pistillata, allowing us to consider the mode of symbiont transmission as an influence on microbiome structure, and also used a deeper sequencing depth to more thoroughly characterize the microbiome.

\section{Endozoicomonas fine-scale specificity resembles} coral-algae and other symbiotic systems

Endozoicomonas appear to be 'core' microbiome members of both $S$. pistillata and $P$. verrucosa, and were detected in $79 \%$ and $85 \%$ of the coral samples, respectively. Although these percentages provide support that Endozoicomonas are core microbiome members, these calculations are dependent on the sequence identity used in the formation of OTUs, which can alter the results (for discussion, see Shade and Handelsman (2012)). Although colonies of $S$. pistillata and $P$. verrucosa both generally associated with a single dominant Endozoicomonas genotype, often several Endozoicomonas OTUs were present, frequently from distinct lineages (Figure 3). This reflects the population structure of the most conspicuous coral endosymbiont, the Symbiodinium algae. Although most coral colonies are dominated by a single Symbiodinium type, several genotypes are generally present (Arif et al., 2014; Quigley et al., 2014). Moreover, in times of heat stress, some corals can undergo a process of 'symbiont shuffling', whereby the current symbionts are replaced with Symbiodinium clades or types that have a higher thermotolerance (Silverstein et al., 2014). It is possible that different Endozoicomonas types are also adapted to different conditions and become abundant at certain times. To test the stability of Endozoicomonas-host associations, we compared 
our Red Sea MED OTUs with Endozoicomonas sequences from a previous study of $S$. pistillata from nearby Red Sea reefs collected in June 2009 (Bayer et al., 2013b) (Figure 3). All of the most abundant sequences from the earlier study had correspondingly abundant MED OTUs (Figure 3), suggesting that the most prevalent Endozoicomonas types maintained their dominance for at least the last 4.5 years. Nevertheless, future studies should explore whether rare Endozoicomonas genotypes become abundant depending on time of day and season, or whether their abundance correlates with the fluctuations of different Symbiodinium clades.

This differential host specificity in Endozoicomonas parallels the specificity seen between Apidae bees and their symbionts. The honeybee, Apis mellifera, and the bumblebee, Bombus spp., both contain two abundant symbionts, Snodgrassella alvi and Gilliamella apicola (Kwong et al., 2014). Despite this similarity, strong host specificity exists for different symbiont strains; for example, S. alvi strains isolated from honeybees could not colonize bumblebees (Kwong et al., 2014). The specificity was driven by host-microbe and microbe-microbe interactions and transmission differences, which may also structure the Endozoicomonas specificity seen in $S$. pistillata and $P$. verrucosa.

\section{Potential functional roles of Endozoicomonas endosymbionts}

Despite the abundance and worldwide distribution of Endozoicomonas in multiple marine organisms, their function is unknown. A suggested role is DMSP breakdown (Raina et al., 2009; Lema et al., 2013; Pike et al., 2013; Vezzulli et al., 2013; Ransome et al., 2014), however, sequenced Endozoicomonas genomes do not contain known DMSP breakdown pathways (Neave et al., 2014). Others have suggested a nutritional symbiosis (La Rivière et al., 2013), interactions with the algal symbiont Symbiodinium (Morrow et al., 2012; Pantos et al., 2015) or roles in producing antimicrobial compounds (Bourne et al., 2008). Although most studies propose that Endozoicomonas are beneficial symbionts, Mendoza et al. (2013) found that cyst-like aggregations of Endozoicomonas were responsible for epitheliocystis in fish and caused mass mortalities. This suggests that different Endozoicomonas genotypes may have disparate roles in their diverse hosts. Genomic sequencing revealed that Endozoicomonas genomes are large ( $>5 \mathrm{Mbs}$ ) and are not streamlined for an obligate endosymbiotic lifestyle, implying they have free-living stages (Neave et al., 2014). The genomes are suggestive of an aerobic heterotrophic lifestyle with the ability to metabolize diverse carbon sources, including amino acids and nucleic acids. The genomes also code for a large number of transport molecules, which they may use to interact with compounds produced by the host or by the Symbiodinium algae.
CARD-FISH revealed the spatial location of Endozoicomonas in the tentacles of $S$. pistillata and in the gastrodermis of $P$. verrucosa, which underlines that different host species show distinct association patterns further supporting that it is (i) a highly intimate relationship and (ii) a highly specialized relationship. Further, the aggregation patterns observed here suggest that Endozoicomonas may utilize metabolic products from other cells within the aggregation, or a component of their metabolism may rely on quorum sensing (Waters and Bassler, 2005). Further, the presence of intratentacular Endozoicomonas aggregates in $S$. pistillata suggests that they may have a role in prey acquisition. In line with this, Schuett et al. (2007) found comparable Endozoicomonas aggregations in the tentacles of a sea anemone. This potentially suggests similar functional roles for some Endozoicomonas genotypes across host species, although further microscopy studies examining the location and arrangement of Endozoicomonas cells in a variety of hosts are required. It should be noted that the rod-shaped Endozoicomonas cells imaged here are distinct from those previously reported for S. pistillata (Bayer et al., 2013b), and the use of CARD-FISH has greatly aided our ability to localize specific cells within highly autofluorescent coral tissues. Altogether, we provide further evidence that Endozoicomonas have an important role in the coral holobiont, however, their precise function remains to be determined.

\section{Conclusions}

Global profiling of microbial community structure in a multi-species framework revealed that Endozoicomonas bacteria are prevalent and often the most abundant prokaryotic symbionts residing intimately within the tissues of two common corals across their global distribution. These differences in Endozoicomonas specificity map onto the different reproductive strategies of the coral hosts. S. pistillata is a brooder and may control the evolution and specificity of its microbiome through the vertical transfer of symbionts, while $P$. verrucosa is a spawner and acquires its prokaryotic symbionts from the water column, thus allowing for greater variability within and less spatial structure across Endozoicomonas genotypes. Our study introduces a coral-bacterial relationship that features a consistent, cosmopolitan, highly aggregated endosymbiont that exhibits some taxonomic variability, and is an important component of the endosymbiotic relationships of the coral holobiont that should be examined with attention to evolutionary and ecological interactions and constraints.

\section{Conflict of Interest}

The authors declare no conflict of interest. 


\section{Acknowledgements}

We thank Ray Dalio for funding the Micronesian expedition and K Hughen, J Ossolinski, C Smith and A Santoro, and the crew of the M/V Alucia for sampling support and the Federated States of Micronesia for collection permits FM12-11-03S and FM12-11-05S. In American Samoa, we are grateful to T Clark of the National Park Service and the Department of Marine and Wildlife Resources for permitting (No. 001) coral collections. In the Maldives, we thank $\mathrm{G}$ Arlotti and the Korallion Laboratory for sampling support and the Ministry of Fisheries and Agriculture for collection permits (No. (OTHR)30-D/INDIV/2013/359). In Australia, we acknowledge $M$ McCulloch and the University of Western Australia for sampling support and the Department of Parks and Wildlife, Western Australia, for permitting (Licence Number SF009558). For sampling in the Red Sea, we are grateful to the CMOR team at KAUST and Red Sea Dream Divers. In Indonesia, we thank P Barber, L Mathar, M Krey, M Dailami, A Fritts-Penniman, the Indonesian Biodiversity Research Center, S-Y Vanson Liu, MZI Noer and A Cahyadi for sampling support, and collection permits from the Wakatobi National Park and the Natural Resources Conservation Center in East Nusa Tenggara, Manokwari (West Papua) and Bali. We acknowledge K Selph of the UH SOEST flow cytometry facility for cell enumeration and J Jennings of Oregon State University for inorganic nutrient analysis. This research was supported by a KAUST-WHOI Post-doctoral Partnership Award to MN and a KAUST-WHOI Special Academic Partnership Funding Reserve Award to CRV and AA. Research in this study was further supported by baseline research funds to CRV by KAUST and NSF award OCE-1233612 to AA. RR was supported by the ct-PIRE Project, Robert Lemelson Fellowship, Graduate Research Award (UCLA), Women Divers Hall of Fame-Sister Fund Conservation Award and a Betty and E. P. Franklin Grant in Tropical Biology and Conservation. Many thanks to E Tambutte and the Centre Scientifique de Monaco (CSM) for provision of coral polyp images. We thank the three anonymous referees for comments that improved this manuscript.

\section{References}

Ainsworth TD, Krause L, Bridge T, Torda G, Raina J-B, Zakrzewski $\mathrm{M}$ et al. (2015). The coral core microbiome identifies rare bacterial taxa as ubiquitous endosymbionts. ISME J 9: 2261-2274.

Amann RI, Binder BJ, Olson RJ, Chisholm SW, Devereux R, Stahl DA. (1990). Combination of 16S rRNA-targeted oligonucleotide probes with flow cytometry for analyzing mixed microbial populations. Appl Environ Microbiol 56: 1919-1925.

Apprill A, Hughen K, Mincer T. (2013). Major similarities in the bacterial communities associated with lesioned and healthy Fungiidae corals. Environ Microbiol 15: 2063-2072.

Apprill A, Marlow HQ, Martindale MQ, Rappé MS. (2012). Specificity of associations between bacteria and the coral Pocillopora meandrina during early development. Appl Environ Microbiol 78: 7467-7475.
Apprill A, Marlow HQ, Martindale MQ, Rappé MS. (2009). The onset of microbial associations in the coral Pocillopora meandrina. ISME J 3: 685-699.

Apprill A, McNally SP, Parsons R, Weber L. (2015). Minor revision to V4 region SSU rRNA 806R gene primer greatly increases detection of SAR11 bacterioplankton. Aquat Microb Ecol 75: 129-137.

Apprill A, Rappé M. (2011). Response of the microbial community to coral spawning in lagoon and reef flat environments of Hawaii, USA. Aquat Microb Ecol 62: 251-266.

Arif C, Daniels C, Bayer T, Banguera-Hinestroza E, Barbrook A, Howe CJ et al. (2014). Assessing Symbiodinium diversity in scleractinian corals via nextgeneration sequencing-based genotyping of the ITS2 rDNA region. Mol Ecol 23: 4418-4433.

Bayer T, Arif C, Ferrier-Pagès C, Zoccola D, Aranda M, Voolstra CR. (2013a). Bacteria of the genus Endozoicomonas dominate the microbiome of the Mediterranean gorgonian coral Eunicella cavolini. Mar Ecol Prog Ser 479: 75-84.

Bayer T, Neave MJ, Alsheikh-Hussain A, Aranda M, Yum LK, Mincer T et al. (2013b). The microbiome of the Red Sea coral Stylophora pistillata is dominated by tissue-associated Endozoicomonas bacteria. Appl Environ Microbiol 79: 4759-4762.

Bourne D, Iida Y, Uthicke S, Smith-Keune C. (2008). Changes in coral-associated microbial communities during a bleaching event. ISME J 2: 350-363.

Caporaso JG, Lauber CL, Walters WA, Berg-Lyons D, Huntley J, Fierer $\mathrm{N}$ et al. (2012). Ultra-highthroughput microbial community analysis on the Illumina HiSeq and MiSeq platforms. ISME $J$ 6: 1621-1624.

Ceh J, van Keulen M, Bourne DG. (2013). Intergenerational transfer of specific bacteria in corals and possible implications for offspring fitness. Microb Ecol 65: 227-231.

Correa H, Haltli B, Duque C, Kerr R. (2013). Bacterial communities of the Gorgonian Octocoral Pseudopterogorgia elisabethae. Microb Ecol 66: 972-985.

Daims H, Brühl A, Amann R, Schleifer K-H, Wagner M. (1999). The domain-specific probe EUB338 is insufficient for the detection of all bacteria: development and evaluation of a more comprehensive probe set. Syst Appl Microbiol 22: 434-444.

De'ath G, Fabricius KE, Sweatman H, Puotinen M. (2012). The 27-year decline of coral cover on the Great Barrier Reef and its causes. Proc Natl Acad Sci USA 109: 17995-17999.

Descombes P, Wisz MS, Leprieur F, Parravicini V, Heine C, Olsen SM et al. (2015). Forecasted coral reef decline in marine biodiversity hotspots under climate change. Glob Chang Biol 21: 2479-2487.

Dinno A. (2015). dunn.test: Dunn's Test of Multiple Comparisons Using Rank Sums. http://cran.r-project. org/package $=$ dunn.test.

Dishaw LJ, Flores-Torres J, Lax S, Gemayel K, Leigh B, Melillo D et al. (2014). The gut of geographically disparate Ciona intestinalis harbors a core microbiota. PLoS One 9: e93386.

Ducklow HW, Mitchell R. (1979). Bacterial populations and adaptations in the mucus layers on living corals. Limnol Oceanogr 24: 715-725.

Edgar RC. (2004). MUSCLE: multiple sequence alignment with high accuracy and high throughput. Nucleic Acids Res 32: 1792-1797. 
Edgar RC, Haas BJ, Clemente JC, Quince C, Knight R. (2011). UCHIME improves sensitivity and speed of chimera detection. Bioinformatics 27: 2194-2200.

Eren AM, Morrison HG, Lescault PJ, Reveillaud J, Vineis JH, Sogin ML. (2014). Minimum entropy decomposition: unsupervised oligotyping for sensitive partitioning of high- throughput marker gene sequences. ISME J 9: $968-979$.

Flot J-F. (2007). champuru 1.0: a computer software for unraveling mixtures of two DNA sequences of unequal lengths. Mol Ecol Notes 7: 974-977.

Flot JF, Magalon H, Cruaud C, Couloux A, Tillier S. (2008). Patterns of genetic structure among Hawaiian corals of the genus Pocillopora yield clusters of individuals that are compatible with morphology. Comptes RendusBiol 331: 239-247.

Folmer O, Black M, Hoeh W, Lutz R, Vrijenhoek R. (1994). DNA primers for amplification of mitochondrial cytochrome c oxidase subunit I from diverse metazoan invertebrates. Mol Mar Biol Biotechnol 3: 294-299.

Forget NL, Juniper KS. (2013). Free-living bacterial communities associated with tubeworm (Ridgeia piscesae) aggregations in contrasting diffuse flow hydrothermal vent habitats at the Main Endeavour Field, Juan de Fuca Ridge. Microbiologyopen 2: 259-275.

Fukatsu T, Koga R, Smith WA, Nikoh N, Sasaki-fukatsu K, Dale C et al. (2007). Bacterial endosymbiont of the slender pigeon louse, Columbicola columbae, allied to endosymbionts of grain weevils and tsetse flies. Appl Environ Microbiol 73: 6660-6668.

Goodson MS, Whitehead LF, Douglas AE. (2001). Symbiotic dinoflagellates in marine Cnidaria: diversity and function. Hydrobiologia 461: 79-82.

Hall VR, Hughes TP. (1996). Reproductive strategies of modular organisms: comparative studies of reefbuilding corals. Ecology 77: 950-963.

Hester ER, Barott KL, Nulton J, Vermeij MJ, Rohwer FL. (2015). Stable and sporadic symbiotic communities of coral and algal holobionts. ISME J 10: 1157-1169.

Hyun D-W, Shin N-R, Kim M-S, Oh SJ, Kim PS, Whon TW et al. (2014). Endozoicomonas atrinae sp. nov., a bacterium isolated from the intestine of a comb pen shell Atrina pectinata. Int J Syst Evol Microbiol 64: 2312-2318.

Jensen S, Duperron S, Birkeland N-K, Hovland M. (2010). Intracellular Oceanospirillales bacteria inhabit gills of Acesta bivalves. FEMS Microbiol Ecol 74: 523-533.

Jessen C, Villa Lizcano JF, Bayer T, Roder C, Aranda M, Wild C et al. (2013). In-situ effects of eutrophication and overfishing on physiology and bacterial diversity of the Red Sea coral Acropora hemprichii. PLoS One 8: e62091.

Kellogg CA. (2004). Tropical Archaea: diversity associated with the surface microlayer of corals. Mar Ecol Prog Ser 273: 81-88.

Keshavmurthy S, Yang S-Y, Alamaru A, Chuang Y-Y, Pichon M, Obura D et al. (2013). DNA barcoding reveals the coral 'laboratory-rat', Stylophora pistillata encompasses multiple identities. Sci Rep 3: 1520.

Kimes NE, Van Nostrand JD, Weil E, Zhou J, Morris PJ. (2010). Microbial functional structure of Montastraea faveolata, an important Caribbean reef-building coral, differs between healthy and yellow-band diseased colonies. Environ Microbiol 12: 541-556.

Knowlton N, Rohwer F. (2003). Multispecies microbial mutualisms on coral reefs: the host as a habitat. Am Nat 162: S51-S62.
Kozich JJ, Westcott SL, Baxter NT, Highlander SK, Schloss PD. (2013). Development of a dual-index sequencing strategy and curation pipeline for analyzing amplicon sequence data on the MiSeq Illumina sequencing platform. Appl Environ Microbiol 79: 5112-5120.

Kwong WK, Engel P, Koch H, Moran NA. (2014). Genomics and host specialization of honey bee and bumble bee gut symbionts. Proc Natl Acad Sci USA 111: 11509-11514.

La Rivière M, Roumagnac M, Garrabou J, Bally M. (2013). Transient shifts in bacterial communities associated with the temperate gorgonian Paramuricea clavata in the Northwestern Mediterranean Sea. PLoS One 8: e57385.

Lema KA, Bourne DG, Willis BL. (2014). Onset and establishment of diazotrophs and other bacterial associates in the early life history stages of the coral Acropora millepora. Mol Ecol 23: 4682-4695.

Lema KA, Willis BL, Bourne DG. (2013). Amplicon pyrosequencing reveals spatial and temporal consistency in diazotroph assemblages of the Acropora millepora microbiome. Environ Microbiol 16: 3345-3359.

Lesser MP, Jarett JK. (2014). Culture dependent and independent analyses reveal no prokaryotic community shifts or recovery of Serratia marcescens in Acropora palmata with white pox disease. FEMS Microbiol Ecol 88: 457-467.

Lesser MP, Mazel CH, Gorbunov MY, Falkowski PG. (2004). Discovery of symbiotic nitrogen-fixing cyanobacteria in corals. Science 305: 997-1000.

Love MI, Huber W, Anders S. (2014). Moderated estimation of fold change and dispersion for RNA-Seq data with DESeq2. Genome Biol 15: 550.

Ludwig W, Strunk O, Westram R, Richter L, Meier H, Yadhukumar et al. (2004). ARB: a software environment for sequence data. Nucleic Acids Res 32: 1363-1371.

McMurdie PJ, Holmes S. (2013). Phyloseq: An R package for reproducible Interactive analysis and graphics of microbiome census data. PLoS One 8: e61217.

McMurdie PJ, Holmes S. (2014). Waste not, want not: why rarefying microbiome data is inadmissible. PLOS Comput Biol 10: e1003531.

Mendoza M, Güiza L, Martinez X, Caraballo X, Rojas J, Aranguren LF et al. (2013). A novel agent (Endozoicomonas elysicola) responsible for epitheliocystis in cobia Rachycentrum canadum larvae. Dis Aquat Organ 106: 31-37.

Morrow KM, Bourne DG, Humphrey C, Botté ES, Laffy P, Zaneveld J et al. (2014). Natural volcanic $\mathrm{CO}_{2}$ seeps reveal future trajectories for host-microbial associations in corals and sponges. ISME J 9: 894-908.

Morrow KM, Moss AG, Chadwick NE, Liles MR. (2012). Bacterial associates of two Caribbean coral species reveal species-specific distribution and geographic variability. Appl Environ Microbiol 78: 6438-6449.

Neave MJ, Michell CT, Apprill A, Voolstra R. (2014). Whole-genome sequences of three symbiotic Endozoicomonas strains. Genome Announc 2: e00802-e00814.

Oksanen J, Blanchet FG, Kindt R, Legendre P, Minchin PR, O'Hara RB et al. (2013). vegan: Community Ecology Package. http://cran.r-project.org/package = vegan.

Pantos O, Bongaerts P, Dennis PG, Tyson GW, HoeghGuldberg O. (2015). Habitat-specific environmental 
conditions primarily control the microbiomes of the coral Seriatopora hystrix. ISME J 9: 1916-1927.

Parada A, Needham DM, Fuhrman JA. (2015). Every base matters: assessing small subunit rRNA primers for marine microbiomes with mock communities, time-series and global field samples. Environ Microbiol 18: 1403-1414.

Park E, Hwang D-S, Lee J-S, Song J-I, Seo T-K, Won Y-J. (2012). Estimation of divergence times in cnidarian evolution based on mitochondrial protein-coding genes and the fossil record. Mol Phylogenet Evol 62: 329-345.

Pike RE, Haltli B, Kerr RG. (2013). Endozoicomonas euniceicola sp. nov. and Endozoicomonas gorgoniicola sp. nov., bacteria isolated from the octocorals, Eunicea fusca and Plexaura sp. Int J Syst Evol Microbiol 63: 4294-4302.

Pinzón JH, Sampayo E, Cox E, Chauka LJ, Chen CA, Voolstra CR et al. (2013). Blind to morphology: Genetics identifies several widespread ecologically common species and few endemics among IndoPacific cauliflower corals (Pocillopora, Scleractinia). J Biogeogr 40: 1595-1608.

Pruesse E, Peplies J, Glöckner FO. (2012). SINA: Accurate high-throughput multiple sequence alignment of ribosomal RNA genes. Bioinformatics 28: 1823-1829.

Quast C, Pruesse E, Yilmaz P, Gerken J, Schweer T, Yarza P et al. (2013). The SILVA ribosomal RNA gene database project: improved data processing and webbased tools. Nucleic Acids Res 41: D590-D596.

Quigley KM, Davies SW, Kenkel CD, Willis BL, Matz MV, Bay LK. (2014). Deep-sequencing method for quantifying background abundances of Symbiodinium types: Exploring the rare Symbiodinium biosphere in reefbuilding corals. PLoS One 9: e94297.

R Development Core Team. (2010), R: A language and environment for statistical computing http://www.Rproject.org.

Raina J-B, Tapiolas D, Willis BL, Bourne DG. (2009). Coralassociated bacteria and their role in the biogeochemical cycling of sulfur. Appl Environ Microbiol 75: 3492-3501.

Ransome E, Rowley SJ, Thomas S, Tait K, Munn CB. (2014). Disturbance to conserved bacterial communities in the cold water gorgonian coral Eunicella verrucosa. FEMS Microbiol Ecol 90: 404-416.

Reshef L, Koren O, Loya Y, Zilber-Rosenberg I, Rosenberg E. (2006). The coral probiotic hypothesis. Environ Microbiol 8: 2068-2073.

Richmond RH. (1987). Energetics, competency, and longdistance dispersal of planula larvae of the coral Pocillopora damicornis. Mar Biol 93: 527-533.

Ritchie KB. (2006). Regulation of microbial populations by coral surface mucus and mucus-associated bacteria. Mar Ecol Prog Ser 322: 1-14.

Robitzch V, Banguera-Hinestroza E, Sawall Y, Al-Sofyani A, Voolstra CR. (2015). Absence of genetic differentiation in the coral Pocillopora verrucosa along environmental gradients of the Saudi Arabian Red Sea. Front Mar Sci 2: 5 .

Roder C, Arif C, Bayer T, Aranda M, Daniels C, Shibl A et al. (2013). Bacterial profiling of White Plague Disease in a comparative coral species framework. ISME J 8: 31-39.

Roder C, Arif C, Daniels C, Weil E, Voolstra CR. (2014). Bacterial profiling of White Plague Disease across corals and oceans indicates a conserved and distinct disease microbiome. Mol Ecol 23: 965-974.

Rodriguez-Lanetty M, Granados-Cifuentes C, Barberan A, Bellantuono AJ, Bastidas C. (2013). Ecological inferences from a deep screening of the complex bacterial consortia associated with the coral. Porites astreoides. Mol Ecol 22: 4349-4362.

Rohwer F, Breitbart M, Jara J, Azam F, Knowlton N. (2001). Diversity of bacteria associated with the Caribbean coral Montastraea franksi. Coral Reefs 20: 85-91.

Rohwer F, Seguritan V, Azam F, Knowlton N. (2002). Diversity and distribution of coral-associated bacteria. Mar Ecol Prog Ser 243: 1-10.

Schloss PD, Westcott SL, Ryabin T, Hall JR, Hartmann M, Hollister EB et al. (2009). Introducing mothur: opensource, platform-independent, community-supported software for describing and comparing microbial communities. Appl Environ Microbiol 75: 7537-7541.

Schmidt-Roach S, Miller KJ, Lundgren P, Andreakis N. (2014). With eyes wide open: A revision of species within and closely related to the Pocillopora damicornis species complex (Scleractinia; Pocilloporidae) using morphology and genetics. Zool J Linn Soc 170: $1-33$.

Schuett C, Doepke H, Grathoff A, Gedde M. (2007). Bacterial aggregates in the tentacles of the sea anemone Metridium senile. Helgol Mar Res 61: 211-216.

Shade A, Handelsman J. (2012). Beyond the Venn diagram: the hunt for a core microbiome. Environ Microbiol 14: 4-12.

Sharp KH, Distel D, Paul VJ. (2012). Diversity and dynamics of bacterial communities in early life stages of the Caribbean coral Porites astreoides. ISME J 6: 790-801.

Sharp KH, Ritchie KB, Schupp PJ, Ritson-Williams R, Paul VJ. (2010). Bacterial acquisition in juveniles of several broadcast spawning coral species. PLoS One 5: e10898.

Shlesinger Y, Goulet TL, Loya Y. (1998). Reproductive patterns of scleractinian corals in the northern Red Sea. Mar Biol 132: 691-701.

Silverstein RN, Cunning R, Baker AC. (2014). Change in algal symbiont communities after bleaching, not prior heat exposure, increases heat tolerance of reef corals. Glob Chang Biol 21: 236-249.

Sogin ML, Morrison HG, Huber JA, Welch DM, Huse SM, Neal PR et al. (2006). Microbial diversity in the deep sea and the underexplored 'rare biosphere'. Proc Natl Acad Sci USA 103: 12115-12120.

Speck MD, Donachie SP. (2012). Widespread Oceanospirillaceae bacteria in Porites spp. J Mar Biol 2012: 1-7.

Stepanauskas R, Sieracki ME. (2007). Matching phylogeny and metabolism in the uncultured marine bacteria, one cell at a time. Proc Natl Acad Sci USA 104: 9052-9057.

Sunagawa S, DeSantis TZ, Piceno YM, Brodie EL, DeSalvo MK, Voolstra CR et al. (2009). Bacterial diversity and White Plague Disease-associated community changes in the Caribbean coral Montastraea faveolata. ISME J 3: 512-521.

Tamura K, Stecher G, Peterson D, Filipski A, Kumar S. (2013). MEGA6: Molecular Evolutionary Genetics Analysis Version 6.0. Mol Biol Evol 30: 2725-2729.

Vezzulli L, Pezzati E, Huete-Stauffer C, Pruzzo C, Cerrano C. (2013). 16SrDNA pyrosequencing of the Mediterranean Gorgonian Paramuricea clavata reveals a link among alterations in bacterial holobiont members, 
anthropogenic influence and disease outbreaks. PLoS One 8: e67745.

Waters CM, Bassler BL. (2005). Quorum sensing: cell-tocell communication in bacteria. Annu Rev Cell Dev Biol 21: 319-346.

Wegley L, Yu Y, Breitbart M, Casas V, Kline DI, Rohwer F. (2004). Coral-associated Archaea. Mar Ecol Prog Ser 273: 89-96.

Whitaker D, Christman M. (2014). clustsig: Significant Cluster Analysis. http://cran.r-project.org/package $=$ clustsig.

Wickham H. (2009). ggplot2: Elegant Graphics for Data Analysis. Springer: New York, NY, USA.

Williams WM, Viner AB, Broughton WJ. (1987). Nitrogen fixation (acetylene reduction) associated with the living coral Acropora variabilis. Mar Biol 94: 531-535.

c) (i) (2) This work is licensed under a Creative Commons Attribution-NonCommercialShareAlike 4.0 International License. The images or other third party material in this article are included in the article's Creative Commons license, unless indicated otherwise in the credit line; if the material is not included under the Creative Commons license, users will need to obtain permission from the license holder to reproduce the material. To view a copy of this license, visit http://creativecommons.org/ licenses/by-nc-sa/4.0/

Supplementary Information accompanies this paper on The ISME Journal website (http://www.nature.com/ismej) 